Ann. Biol. anim. Bioch. Biophys., 1979, 19 (4 A), 1123-1130.

\title{
Effet de la sécrétion biliaire sur la digestion chez le porc
}

\author{
par T. CORRING, Catherine JUSTE, C. SIMOES-NUNES, D. BOURDON * \\ avec la collaboration technique de Anne-Marie GUEUGNEAU, J. P. HAUDUCGUR, A. ROGER, \\ R. LEVREL, G. DUCHASTEL \\ Laboratoire de Physiologie de la Nutrition \\ * Station de Recherches sur l'Elevage des Porcs \\ I.N.R.A., 78350 jouy en josas.
}

Summary. Effect of biliary secretion on digestion in the pig.

The apparent digestibility of a diet was studied either after ligature of the bile duct or after discontinuous removal of bile secretion in the growing pig. Feces and urine were collected during a 10-day period in bile duct-ligated pigs, and over three 5-day periods in bile-fistulated pigs. The latter were subjected to three treatments each over the three periods : 1) re-entry of bile into the duodenum ; 2) introduction of a salt mixture ( $\mathrm{Na}$ : $\left.150 \mathrm{meq} / \mathrm{l} ; \mathrm{Cl}: 120 \mathrm{meq} / \mathrm{l} ; \mathrm{HCO}_{3}: 40 \mathrm{meq} / \mathrm{l}\right)$; 3) no re-entry of either bile or the salt mixture. Apparent energy digestibility was mainly affected after ligature of the bile duct (-31.6 p. 100) or after removal of bile from the intestinal lumen ( -23 p. 100). This decrease was essentially due to a decrease in the total lipid apparent digestibility ( $-53 \mathrm{p}$. 100 after bile duct ligation and -31.7 p. 100 after bile removal). Apparent nitrogen and energy derived from carbohydrates digestibilities were unaffected. In the fistulated pigs the intraduodenal introduction of the salt mixture did not improve decreased apparent digestibilities.

\section{Introduction.}

Le rôle de la bile dans la lipolyse des lipides alimentaires ou/et l'absorption des produits issus de cette hydrolyse a été précisé par de nombreux auteurs (Heersma et Annegers, 1948a ; Pessoa, Kim et Ivy, 1953 ; Holt, 1972). Cependant peu de travaux portent sur l'étude du bilan de l'action de la sécrétion biliaire dans la digestion (Heersma et Annegers, 1948b; Cohen, 1961). Le but du présent travail a été d'estimer ce bilan chez le Porc, par la mesure de la digestibilité apparente et de la rétention. Contrairement aux espèces classiquement utilisées : le chien et le rat, et plus récemment le mouton, le Porc offre l'avantage de présenter une physiologie digestive très semblable à celle de l'homme. Il peut être proposé comme modèle d'animal expérimental dans les recherches visant à préciser nos connaissances sur la digestion chez l'homme. 


\section{Matériel et méthodes.}

Deux techniques on été appliquées : la première (ligature du canal cholédoque) a conduit à étudier l'effet à long terme et la seconde (dérivation de la sécrétion biliaire) l'effet à court terme de l'élimination de la bile de la lumière intestinale, sur la digestibilité apparente ef la rétention d'un régime riche en lipides.

Des porcs de race Large White, mâles castrés, de poids vif moyen de $55 \mathrm{~kg}$ avant intervention chirurgicale, ont été utilisés. La composition du régime expérimental est rapportée dans le tableau 1. Les pourcentages de cellulose et de vermiculite sont élevés de façon à diluer l'apport énergétique dû à la haute teneur du régime en huile d'arachide.

\section{TABLEAU 1}

Composition du régime expérimental

\begin{tabular}{|c|c|c|c|}
\hline Composants & $\mathrm{g} / 100 \mathrm{~g}$ & p. 100 matière sèc & \\
\hline 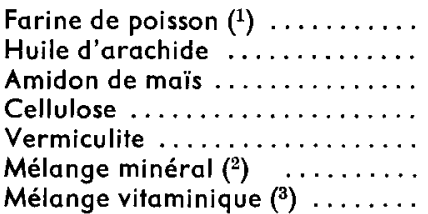 & $\begin{array}{l}23 \\
21 \\
20 \\
15 \\
17,5 \\
2,5 \\
1\end{array}$ & $\begin{array}{l}\text { Matière sèche } \\
\text { Protéines }(N \times 6,25) \\
\text { Energie }(\mathrm{kcal} / \mathrm{kg})\end{array}$ & $\begin{array}{l}93,8 \\
15,8 \\
4700\end{array}$ \\
\hline
\end{tabular}

(1) 68,6 p. 100 protéines.

(2) En p. 100 du régime : phosphate dicalcique, 1,25; craie broyée, 0,19 ; chlorure de sodium, 0,38 ; chlorure de potassium, 0,50 ; carbonate de magnésie, 0,06 ; oligo-éléments en $\mathrm{mg} / \mathrm{kg}$ aliment : $\mathrm{FeSO}_{4}, 7 \mathrm{H}_{2} \mathrm{O}, 480$; $\mathrm{MnSO}_{4}, \mathrm{H}_{2} \mathrm{O}, 144 ; \mathrm{CuSO}_{4}, 5 \mathrm{H}_{2} \mathrm{O}, 120 ; \mathrm{ZnSO}_{4}, \mathrm{H}_{2} \mathrm{O}, 270 ; \mathrm{KI}, 0,06$.

$\left.{ }^{3}\right) \mathrm{En} \mathrm{mg/kg}$ aliment: acétate de rétinol (50 000 UI), 100 ; cholécalciférol (100000 UI), 10 ; $\alpha$-tocophérol ( $250 \mathrm{UI}), 160$; vitamin $\mathrm{K}, 4.4$; vitamine $B_{1}, 2,2$; vitamine $B_{6}, 2,6$; riboflavine, 6,2 ; acide nicotinique, 22 ; acide folique, 1,6 ; vitamine $B_{12}(500 \mathrm{mg} / \mathrm{kg}), 66$; inositol, 400 ; biotine, 12 ; pantothénate de calcium, 20 ; choline (50 p. 100), 2600 ; acide paraminobenzoïque, 44.

\section{Schéma expérimental.}

1) Ligature du Canal cholédoque. - Huit porcs ont été utilisés. Après adaptation au régime et à la cage à métabolisme pendant 8 jours, 4 porcs ont subi une ligature du canal cholédoque ef les 4 autres animaux une laparotomie latérale (animaux témoins). Après une période de récupération de 14 jours, la digestibilité apparente ef la rétention ont été mesurées sur une période de 10 jours. Les porcs ont reçu la même quantité d'aliment (méthode du paired-feeding de Mitchell, 1930) et les refus ont été mesurés après chaque repas ( 3 par jour). A la fin de la collecte des fèces ef urines, les animaux ont été abattus ; le foie a été pesé et un échantillon de sang systémique prélevé en vue d'une détermination du cholestérol libre ef total (Watson, 1960).

Technique chirurgicale de la ligature : Les porcs sont mis à jeun 24 h avant l'anesthésie. Après une laparotomie latérale droite, 2 ligatures sont placées, proches l'une de l'autre, autour du canal cholédoque à environ $1 \mathrm{~cm}$ du duodénum. Le canal est sec- 
tionné entre les 2 ligatures. Les animaux témoins subissent la même intervention excepté la pose des ligatures ef la section du canal.

2) Dérivation discontinue de la bile chez l'animal fistulé. - Six porcs ont été munis de fistules permanentes du canal cholédoque ef du duodénum (Juste, Corring et Breant, 1979), et placés en cage à métabolisme. Après une période de récupération post opératoire de 10 jours, les animaux donf la bile recueillie était réintroduite en permanence dans le duodénum ont été chacun, soumis à 3 traitements sur 3 périodes de 8 jours : a) Réintroduction de bile dans le duodénum, b) Introduction d'une solution d'électrolytes, $c$ ) Absence totale de réintroduction de bile ou d'électrolyłes. Un schéma récapitulatif de cette expérimentation est donné dans le tableau 2. Un tel schéma pré-

TABLEAU 2

Dérivation discontinue de la sécrétion biliaire : Schéma expérimental

\begin{tabular}{|c|c|c|c|c|c|c|}
\hline Pore & 1 & 2 & 3 & 4 & 5 & 6 \\
\hline $\begin{array}{l}1 \text { re période } \ldots \ldots \ldots \\
2^{\mathrm{e}} \text { période } \ldots \ldots \ldots \\
3^{\mathrm{e}} \text { période } \ldots \ldots \ldots\end{array}$ & $\begin{array}{l}\mathrm{B}\left({ }^{1}\right) \\
O\left({ }^{2}\right) \\
S\left({ }^{3}\right)\end{array}$ & $\begin{array}{l}O \\
S \\
B\end{array}$ & $\begin{array}{l}\mathrm{S} \\
\mathrm{O} \\
\mathrm{B}\end{array}$ & $\begin{array}{l}\text { B } \\
\mathbf{S} \\
\mathbf{O}\end{array}$ & $\begin{array}{l}O \\
B \\
S\end{array}$ & $\begin{array}{l}\text { S } \\
\text { B } \\
\text { O }\end{array}$ \\
\hline
\end{tabular}

(1) Réintroduction de bile dans le duodénum. 8 jours.

(2) Absence de réintroduction de bile ou d'électrolyłes. 8 jours.

(3) Introduction d'une solution d'électrolytes dans le duodénum ( $\mathrm{Na}: 150 \mathrm{meq} / \mathrm{l} ; \mathrm{Cl}: 120 \mathrm{meq} / \mathrm{l}$; $\mathrm{HCO}_{3}: 40$ meq/l) 8 jours.

vient tout effet relatif à l'ordre séquentiel des traitements et permet de comparer sur le même animal la digestibilité apparente et la rétention en absence ou en présence de bile ou d'électrolytes dans la lumière intestinale. La collecte des fèces et urines a été effectuée en cours des 5 derniers jours de chaque période.

La réintroduction de bile a été réalisée au moyen d'une pompe péristaltique à différentes vitesses selon l'heure de la journée (Juste, Corring et Bréant, 1979 ; Juste ef Corring, 1979). Les modalités de l'introduction intraduodénale de la solution d'électrolytes sont identiques. Les porcs ont reçu la même quantité d'aliment (méthode du paired-feeding de Mitchell, 1930) et les refus ont été mesurés après chaque repas (3 par jour).

Mesure de la digestibilité apparente ef de la rétention : Les modalifés expérimentales des collectes de fèces et d'urines ont été décrites par Henry et Rérat (1966). L'énergie de l'aliment et des fèces a été déterminée à l'aide d'un calorimètre adiabatique ( ${ }^{1}$ ).

Les critères de mesure sont les digestibilités apparentes de la matière organique, de l'énergie tolale, de l'azote et des lipides totaux solubles ainsi que les rétentions de l'azote ef de l'énergie. La détermination des lipides totaux dans le régime ef les fèces a été réalisée selon la technique de Folch, Lees et Sloane Stanley (1957). Les calculs de la digestibilité apparente et de la rétention ont été faits selon les formules

(1) Calorimètre Gallenkamp, OSI France, Paris. 
Digestibilité apparente :

$$
\frac{\text { Absorbé }}{\text { Ingéré }} \times 100=\frac{\text { Ingéré }- \text { Excrété dans les fèces }}{\text { Ingéré }} \times 100 \text {. }
$$

Rétention :

$\frac{\text { Retenu }}{\text { Absorbé }} \times 100=\frac{\text { Ingéré }- \text { Excrété dans les fèces }- \text { Excrété dans l'urine }}{\text { Ingéré }- \text { Excrété dans les fèces }} \times 100$.

D'autre part, la digestibilité apparente de l'énergie provenant des glucides a été estimée selon la formule :

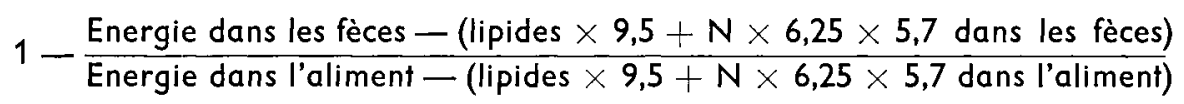

où 9,5 et 5,7 représentent respectivement l'énergie digestible des lipides ef des matières azotées totales (kcal/g ; Nehring, Beyer et Hoffman, 1972).

Par ailleurs, tous les porcs ont été pesés le premier et dernier jour de chaque période de collecte.

\section{Résultats.}

1) Ligature du canal cholédoque.

Les animaux ont été abattus 30 jours après la ligature du canal cholédoque. Un des quatre porcs ligaturés est mort au cours de la période de collecte des fèces et urines. Les trois autres avaient développé un important ictère et les mesures post-mortem montrent que le rapport poids du foie/poids vif est supérieur chez ces animaux à celui observé chez les témoins $(33 \mathrm{~g} / \mathrm{kg} \pm 1,8$ contre $16 \mathrm{~g} / \mathrm{kg} \pm 1,1)$. De plus chez les porcs ligaturés, les valeurs du cholestérol sanguin total et libre sont supérieures à celles déterminées chez les témoins : respectivement $2,18 \mathrm{~g} / \mathrm{l}$ contre $1,41 \mathrm{~g} / \mathrm{l}$ et $0,30 \mathrm{~g} / \mathrm{l}$ contre $0,18 \mathrm{~g} / \mathrm{l}$. Le gain de poids moyen a été de $337 \mathrm{~g} \pm 145 \mathrm{chez}$ les animaux ligaturés et $662 \mathrm{~g} \pm 63$ chez les témoins $(P<0,01)$.

Les valeurs des digestibilités apparentes et des rétentions sont rapportées dans le tableau 3.

Si l'on se réfère aux mesures effectuées chez les animaux témoins, on constate que la ligature du canal cholédoque a entraîné une diminution de 18,9 p. 100 de la digestibilité apparente de la matière organique. Mais la suppression de bile a principalement affecté la digestibilité apparente de l'énergie qui diminue de 31,6 p. 100. Si la digestibilité apparente calculée de l'énergie issue des glucides n'a pas été modifiée par la ligature du canal cholédoque, la digestibilité apparente des lipides totaux a diminué de 52,3 p. 100. En ce qui concerne celle de l'azote, elle n'est pas modifiée par l'élimination de la bile de la lumière duodénale.

La rétention de l'azote est semblable dans les 2 groupes d'animaux tandis que l'on note une diminution significative de la rétention de l'énergie chez les porcs ligaturés (-4 p. 100). 
TABLEAU 3

Digestibilités apparentes du régime mesurées

du $15^{\mathrm{e}}$ au $25^{\mathrm{e}}$ jour après ligature du canal cholédoque chez le porc

\begin{tabular}{|c|c|c|c|}
\hline & $\begin{array}{l}\text { Animaux } \\
\text { ligaturés }\end{array}$ & $\begin{array}{l}\text { Animaux } \\
\text { témoins }\end{array}$ & $\begin{array}{c}\text { Signification } \\
\text { statistique }\end{array}$ \\
\hline 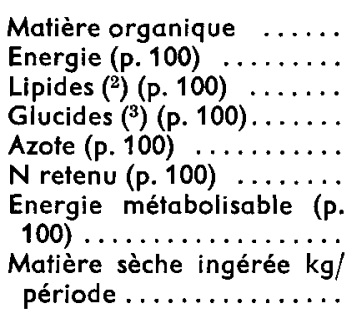 & $\begin{array}{l}52,8 \pm 0,8(1) \\
46,4 \pm 1,2 \\
38,3 \pm 2,8 \\
43,2 \pm 1,9 \\
77,4 \pm 0,6 \\
32,4 \pm 9,0 \\
92,3 \pm 0,3 \\
13,0\end{array}$ & $\begin{array}{l}65,1 \pm 1,3 \\
67,9 \pm 2,0 \\
80,3 \pm 4,0 \\
45,0 \pm 2,6 \\
78,4 \pm 1,8 \\
49,0 \pm 12,3 \\
96,0 \pm 0,5 \\
13,6\end{array}$ & $\begin{array}{c}P<0,001 \\
P<0,001 \\
P<0,001 \\
N S \\
N S \\
\quad N S \\
P<0,001\end{array}$ \\
\hline
\end{tabular}

(1) Moyenne \pm SEM.

(2) Lipides totaux.

$\left({ }^{3}\right)$ Energie provenant des glucides. Analyse statistique : analyse de variance, test t de Student.

\section{2) Dérivation discontinue de la sécrétion biliaire.}

La dérivation de la bile hors de la lumière intestinale se traduit en premier lieu par une perte de poids des animaux sur toute la période de collecte des fèces et urines $(-500 \mathrm{~g})$. Par contre, il y a gain de poids lorsque la bile est réintroduite $(+2200 \mathrm{~g})$ ef lorsque une solution d'électrolytes est introduite dans le duodénum $(+1200 \mathrm{~g})$.

\section{TABLEAU 4}

Digestibilités apparentes du régime après dérivation discontinue de la sécrétion biliaire, chez le porc

\begin{tabular}{|c|c|c|c|c|}
\hline & + bile $\left({ }^{1}\right)$ & - bile $\left({ }^{2}\right)$ & + électrolytes $\left({ }^{3}\right)$ & $\begin{array}{l}\text { Signification } \\
\text { statistique }\end{array}$ \\
\hline 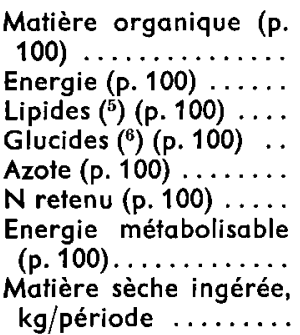 & $\begin{array}{l}58,1 \pm 4,8\left({ }^{4}\right) a \\
56,1 \pm 6,9 a \\
62,2 \pm 10,2 a \\
40,0 \pm 8,5 a \\
75,2 \pm 2,5 a \\
39,0 \pm 7,6 a \\
94,3+1,3 a \\
\quad 7,0\end{array}$ & $\begin{array}{l}50,6 \pm 5,3 b \\
43,2 \pm 6,3 b \\
42,5 \pm 16,0 b \\
38,8 \pm 10,7 a \\
74,3 \pm 2,6 a \\
18,6 \pm 12,9 b \\
91,6 \pm 2,7 a \\
6,5\end{array}$ & $\begin{array}{c}51,4 \pm 3,4 b \\
45,2 \pm 4,3 b \\
44,2 \pm 13,4 b \\
40,3 \pm 6,8 a \\
75,8 \pm 2,9 a \\
36,7 \pm 6,8 a \\
93,5 \pm 0,9 a \\
7,0\end{array}$ & $\begin{array}{c}P<0,05 \\
P<0,01 \\
P<0,01 \\
N S \\
N S \\
P<0,05 \\
\text { NS }\end{array}$ \\
\hline
\end{tabular}

(1) Réintroduction de bile dans le duodénum. $\left({ }^{2}\right)$ Absence de réintroduction de bile ou d'électrolytes. $\left({ }^{3}\right)$ Introduction d'électrolytes dans le duodénum. $\left({ }^{1}\right),\left({ }^{2}\right)$ ef $\left({ }^{3}\right)$ Collectes fèces et urines durant 5 jours.

$\left(^{(4)}\right.$ Moyenne \pm SEM. $\left(^{5}\right)$ Lipides totaux. $\left(^{6}\right)$ Energie provenant des glucides. Analyse statistique basée sur un schéma plurifactoriel linéaire. La signification statistique se rapporte à la différence entre traitements : les moyennes sur une même ligne non suivies de la même lettre sont significativement différentes au $P$ indiqué. 
Les résultats des digestibilités apparentes et des rétentions sont rapportés dans le tableau 4. Toutes les données ont été statistiquement interprétées selon le schéma plurifactoriel linéaire :

$$
Y_{i j k}=\mu+\alpha_{i}+\beta_{j}+\gamma_{k}+e_{i j k}
$$

où $\mu$ exprime l'effet global, $\alpha$ l'effet animal, $\beta$ l'effet période, $\gamma$ l'effet traitement et e l'effet résiduel. Ce schéma a permis de montrer l'absence de l'effet période quelle que fut la séquence des traitements et l'absence de tout effet animal. Seul un effet du traitement est mis en évidence. Si l'on se réfère aux valeurs obtenues au cours de la période de réintroduction de la bile, la dérivation de cette sécrétion de la lumière intestinale entraîne une diminution de la digestibilité apparente de la matière organique (-12,9 p. 100), de l'énergie totale (-23 p. 100), des lipides totaux ( $-31,7$ p. 100) et de la rétention azotée (-52,3 p. 100). Par contre, ni la digestibilité apparente de l'azote, ni celle calculée de l'énergie provenant des glucides n'ont été modifiées. L'introduction intestinale de la solution d'électrolytes conduit à des digestibilités apparentes non statistiquement différentes de celles obtenues chez l'animal privé de sécrétion biliaire, excepté la rétention azotée dont la valeur est identique à celle observée chez le porc dont la bile est retournée au niveau duodénal.

\section{Discussion.}

Il importe tout d'abord de noter que dans les deux expérimentations ef chez les animaux témoins, la digestibilité apparente de la matière organique a été relativement faible. Cela peut être expliqué par les pourcentages élevés dans le régime de cellulose ef de vermiculite, composants peu ou non digestibles, destinés à diluer l'apport énergétique dû à la forte teneur en lipides alimentaires.

La ligature du canal cholédoque ou la dérivation de la sécrétion biliaire hors de la lumière intestinale entraîne une diminution de la digestibilité apparente de l'énergie totale, essentiellement due à l'accroissement de la quantité de lipides dans les fèces. En effet, la digestibilité apparente de l'azote et celle calculée de l'énergie provenant des glucides n'ont pas été modifiées. Il apparaît donc que la bile est spécifiquement impliquée dans l'utilisation digestive des lipides alimentaires. Un tel résultat confirme les observations réalisées chez l'homme après résection de la partie distale de l'intestin grêle (Hardison et Rosenberg, 1967 ; Poley et Hofmann, 1976) ef chez l'animal déficient en bile (Heersma et Annegers, 1948b; Pessoa, Kim et Ivy, 1953 ; Cohen, 1961).

Harkins ef al. (1965) montrent que les graisses saturées sont moins bien utilisées que les graisses insaturées en cas de déficiences biliaires. Le régime expérimental utilisé dans le travail rapporté ici contient un fort pourcentage de graisses non saturées ; il s'ensuit que les résultats obtenus ne permettent aucune application à d'autres graisses telles que le lard ou le saindoux, par exemple.

Si ces résultats présentent néanmoins l'intérêt de quantifier, pour la première fois chez le porc, l'effet de la sécrétion biliaire dans l'utilisation digestive d'un aliment renfermant essentiellement des lipides insaturés, les limites de la méthodologie utilisée ne peuvent permettre de répondre à un certain nombre de questions. La mesure de 
la digestibilité apparente donne des informations sur le rôle de la bile dans le processus général : lipolyse + absorption, mais ne renseigne pas sur l'étape de digestion affectée par l'absence de la bile. Selon certains auteurs, la bile serait peu impliquée dans l'hydrolyse enzymatique des triglycérides alimentaires; la lipolyse peut avoir lieu en l'absence complète de bile (Heath et Morris, 1963 ; Knoebel et Ryan, 1963 ; Porter, Saunders et Tytgat, 1971). Cependant la vitesse de la lipolyse est augmentée en présence de bile (Holt, 1972). En revanche, la solubilisation micellaire des produits de l'hydrolyse serait principalement affectée par l'absence de bile (Clark ef Holt, 1969) ; ainsi une diminution de l'absorption de ces produits pourrait expliquer l'accroissement de la quantité des lipides fécaux (Borgstrom, 1953 ; Heath ef Hill, 1969 ; Gallacher Webb et Dawson, 1965). En cas de déficience biliaire, on peut également poser la question du site d'absorption des lipides (jejunum, ileum) ou du rôle, si elle existe, de l'absorption dans le gros intestin. Quelle est la part prise par la microflore dans l'hydrolyse de triglycérides arrivant intacts dans la partie distale du tractus digestif ? Chez le rat dont la sécrétion pancréatique a été éliminée par ligature du canal excréteur (Corring, Moreau et Ducluzeau, 1979) il a été démontré que la microflore peut compenser l'absence des enzymes protéolytiques pancréatiques et hydrolyser la protéine alimentaire. D'autre part on ne peut éliminer l'éventualité de changements de la microflore intestinale après suppression ou dérivation de la sécrétion biliaire. De telles modifications peuvent entraîner une dégradation des lipides d'origine bactérienne d'où une diminution de leur digestibilité apparente chez les animaux privés de bile.

Bien que la digestibilité apparente de l'azote n'ait pas été modifiée par l'absence de bile, ce qui confirme les observations faites chez l'homme (Fowweather, 1926 ; Thaysen, 1926-1927) ef le chien (Heersma ef Annegers, 1948b), la technique utilisée rendant compte d'un bilan ne permet pas de préciser si la vitesse d'absorption des produits d'hydrolyse a été ou non affectée. Enfin, la quantité d'azote retenue après absorption a été nettement diminuée lorsque la bile est absente de la lumière intestinale mais non modifiée lorsque la bile ou une solution d'électrolytes ont été introduites dans le duodénum. Aucune explication ne peut être donnée actuellement sur ce dernier point.

Reçu en février 1979.

Accepté en mars 1979.

Remerciements. - Nous remercions Mlle Noelle Bourgeaux et M. G. Durand (Station de Recherches de la Nutrition, I. N. R. A.) pour les déterminations du cholestérol.

\section{References}

BORGSTROM B., 1953. On the mechanism of the intestinal fat absorption in the rat. V. The effect of bile diversion on fat absorption in the rat. Acto physiol. scand,. 28, 279-286.

CLARK S. B., HOLT P. R., 1969. Inhibition of steady-state intestinal absorption of long-chain triglyceride by medium chain triglyceride in the unanaesthetized rat. J. clin. Invest., 48, 2235-2243.

COHEN B. J., 1961. Fat excretion in dogs lacking both bile and pancreatic juice. Proc. Soc. exp. Biol. Med., 107, 40-42. 
CORRING T., MOREAU Ch., DUCLUZEAU R., 1979. Comparative apparent digestibility of casein in holexenic, axenic and Clostridium bifermentans monoassociated rats. Amer J. clin. Nutr. 32 (in press).

FOLCH J., LEES M., SLOANE STANLEY G. H., 1957. A simple method for the isolation and purification of tolal lipids from animal tissues. J. biol. Chem., 226, 497-509.

FOWWEATHER F. S., 1926. The determination of the amount and the composition of the fat in faeces. II. The composition of the fat of the faeces of the normal adult as ascertained by the "wet" method, together with some results in certain pathological conditions. Brit. J. exper. Path., 7, 15-21.

GALLACHER N., WEBB J., DAWSON A. M., 1965. The absorption of $\left({ }^{14} \mathrm{C}\right)$ oleic acid and $\left({ }^{14} \mathrm{C}\right)$ triolein in bile fistula rat. Clin. Sci., 29, 73-82.

HARDISON W. G. M., ROSENBERG I. H., 1967. Bile salt deficiency in the steatorrhea following resection of the ileum and proximal colon. N. Engl. J. Med., 277, 337-343.

HARKINS R. W., HAGERMAN L. M., SARETT H. P., 1965. Absorption of dietary fats by the Rat in cholestyramine-induced steatorrhea. J. Nutrition, 87, 85-92.

HEATH T. J., MORRIS B., 1963. The role of bile and pancreatic juice in the absorption of fat in ewes and lambs. Br. J. Nutr., 17, 465-474.

HEATH T. J., HILL L. N., 1969. Dietary and endogenous long-chain fatty acids in the intestine of sheep, with, an appendix on their estimation in feeds, bile and faeces. Austr. J. Biol. Sci., 22, 1015-1029.

HEERSMA J. R., ANNEGERS J. H., 1948a. Effect of bile preparations on fat absorption in bile fistula dogs. Proc. Soc. exp. Biol. Med., 67, 339-341.

HEERSMA J. R., ANNEGERS J. H., 1948b. Effect of bile diversion on fecal fat and nitrogen excretion. Am. J. Physiol., 153, 143-147.

HENRY Y., RÉRAT A., 1966. Utilisation des pommes de terre déshydratées et fraîches dans l'alimentation du porc en croissance en comparaison avec l'orge. Ann. Zootech., 15, $231-251$.

HOLT P. R., 1972. The roles of bile acids during the process of normal fat and cholesterol absorption. Arch. infern. Med., 130, 574-583.

JUSTE C., CORRING T., BREANT Ph., 1979. Excrétion biliaire chez le porc : niveau et réponse au repas. Ann. Biol. anim. Bioch. Biophys., 19, 79-90.

JUSTE C., CORRING T., 1979. Effet d'une interruption et de restitutions partielles du recyclage entérohépatique sur le niveau de l'excrétion biliaire chez le porc. Ann. Biol. anim. Bioch. Biophys., 19, 405-412.

KNOEBEL K. K., RYAN J. M., 1963. Digestion and mucosal absorption of fat in normal and bile deficient dogs. Am. J. Physiol., 204, 509-514.

MITCHELL H. H., 1930. The paired-feeding method : its value and limitations in live-stock experimentation. Proc. Am. Soc. Anim. Prod., 63-73.

NEHRING K., BEYER M., HOFFMAN B., 1972. Fuftermiffel tabellenwerk Veb. Deutscher Landwirtschaftsverlag, Berlin, Pp. 261.

PESSOA V. C., KIM K. S., IVY A. C., 1953. Fat absorption in absence of bile and pancreatic juice. Am. J. Physiol., 174, 209-218.

POLEY J. R., HOFMANN A. F., 1976. Role of fat maldigestion in pathogenesis of steatorrhea in ileal resection. Gastroenterology, 71, 38-44.

PORTER H. P., SAUNDERS D. R., TYTGAT C., 1971. Fat absorption in bile fistula man : a morphological and biochemical study. Gastroenterology, 60, 1008-1019.

THAYSEN T. E., 1926-1927. On steatorrhea. Acta med. scand. Supplem. 16, 384-396.

WATSON D., 1960. A simple method for the determination of serum cholesterol. Clin. chim. Acta, 5, 637-643. 